\title{
MultiView Program Status: Data Standards for the Integrated Digital Environment
}

\author{
Richard L. Engwall ${ }^{1}$, and John W. Reber ${ }^{2}$ \\ ${ }^{\prime}$ RLEngwall \& Associates, USA, ${ }^{2}$ Trident Systems Inc,USA, rlengwall@aol.com
}

\begin{abstract}
The MultiView (MV) Program is a USA Research \& Development project, which focuses on developing data standards for the integrated digital environment. The aim is to achieve a high degree of interoperability of the Information Technology (IT) systems for complex engineer-to-order systems, products and processes over their life cycle.

Project objectives are to use available software and standards, provide a single schema for seamless integration of the data sets and to develop a framework for data access and communication over the life cycle of the products as well as the systems.
\end{abstract}

\section{INTRODUCTION}

The MultiView (MV) Program is a multi-year USA congressionally mandated Research \& Development project, managed by the U.S. Army Logistics Integration Agency, developed by Trident Systems Inc. as prime contractor and Concurrent Technologies Corporation and RLEngwall \& Associates as subcontractors. MultiView's focus is on developing data standards for the integrated digital environment. The program start was July 2000; program completion is dependent upon obtaining year-to-year available funding.

Enterprises that acquire and sustain complex modern weapons systems face unprecedented challenges in containing costs while taking their systems through concept, design, development, deployment, and retirement. The schema and associated data set required for specifying, developing, operat- 
ing, maintaining, and disposing of such systems is extremely large and involves myriad subtle relationships among seemingly disparate domains. Added to this complex reality, contractors and program offices must develop, deliver, and manage systems meeting aggressive readiness requirements and shifting mission objectives within stringent budget constraints. Affordability has become as important as mission performance when developing and sustaining such systems.

Similarly, product customization to satisfy specialized customer requirements, time-to-market, and affordability have become as important to the industrial world as product performance is for commercial complex systems. Commercial companies are increasingly operating in a virtual extended enterprise environment striving to share selective information with their myriad of disparate distributed stakeholders. Therefore the industrial approach to solving these problems through standards and partnering activities can be used as a model for the Department of Defense (DOD) and the MV Program.

There is significant commonality of interest between DOD and U.S. industry in trying to achieve a high degree of interoperability of their Information Technology (IT) systems for complex engineer-to-order systems, products and processes over their life cycle. Success in so doing minimizes the number and cost of transactions and results in a lean more affordable operating mode for all involved. The path to this objective (and the specific objective) of the MV program involves the use to maximum extent possible of:

- Existing commercial enterprise software and standards

- A single schema for seamless integration of broad and varied data sets

- A framework or architecture for the communication and access to this data, over the life cycle of the system or product involved

\section{DESCRIPTION OF PROBLEM}

Military weapon system program offices must be able to define technical or fiscal metrics to assess the Total Ownership Cost (TOC) of their systems. Traditional system acquisition and life cycle management practices include the use of automated tools for modeling and simulation, configuration management, and supply support systems to create and manage technical data for systems both in development and in the field. However, each tool uses its own data representation and storage mechanism causing major problems in communicating between systems.

With a few exceptions, mostly in the commercial industrial world, no real interoperability exists among tools, even for exchanging data concerning the same technical area of the system. Human operators most often re-enter data for each tool employed in the process. Interoperability problems grow with 
automated support. As program management offices use advanced process modeling and planning techniques, and work with complex sets of data across multiple databases, as shown in Fig. 1 the interoperability problem multiplies. This presents a significant and growing challenge to programs to effectively integrate complex system data.

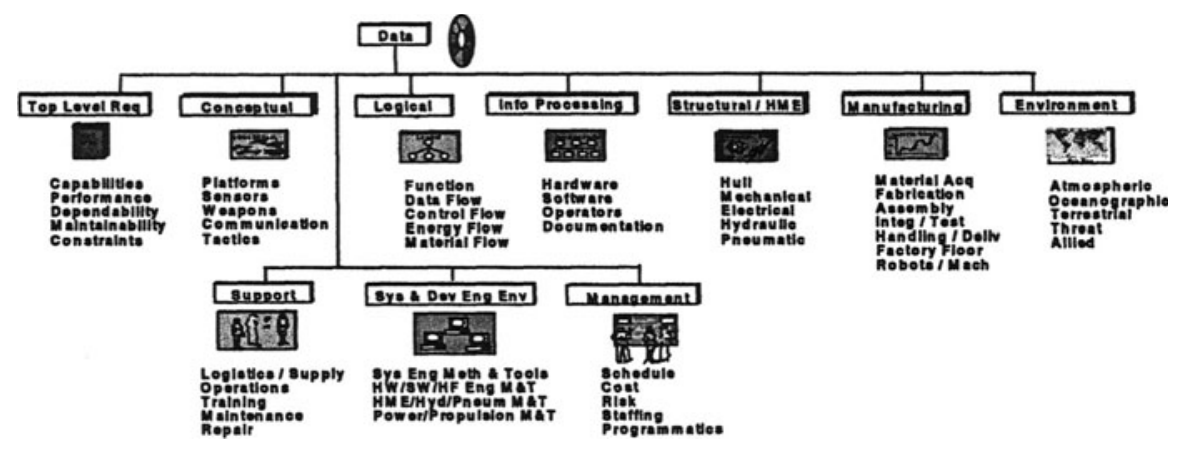

Figure 1 Product Life Cycle Multiple Domain Information Needs

In the commercial industrial world "best of breed" enterprise integration interoperability solutions are based on achieving as much integration as possible through use of:

- Applying Business-Process Re-engineering (BPR) to simplify business processes;

- Tailoring business streams to separate basic/core, options, and unique processes;

- Developing a structured information systems framework/application integration middleware solution focusing on a single call data communication employing a common objective model using standards accepted by the international community or de facto standards such as OMG-CORBA-2, (1999), CORBA-bus, to abstract data at the data interface business object level not the database/schema level;

- Deploying selective use of a portfolio of international information systems standards such as STEP, (ISO IS 10303, 1999), Web-based XML (2000, 2001), and ANSI X12 EDI data transfer/presentation solutions (2000);

- Deploying Commercial-off-the-Shelf (COTS) application solutions for production and management processes such as: ERP, PDM, MES, SCM, CRM, etc. 
Application of these individual company COTS tools solutions just further exacerbates the overall enterprise interoperability problem. Most of the present enterprise interoperability successes have been with managementrelated textual business processes and not with the technical processes involving systems engineering, product and factory modeling and simulations, CAE, CAD, CAM and product life cycle support processes. The MV Program's prime focus is to address these technical business process areas and to integrate the technical information with the management business process areas.

\section{3}

\section{APPROACH TO PROBLEM SOLUTION}

Meeting the challenge to effectively integrate complex system data is a key to ensuring that complex systems (such as the Abrams Tank, the Navy's 21 st Century Destroyer, and the Joint Strike Fighter, and commercial complex systems such as wide-body airplanes, automobiles, trucks, ships, trains, offshore oil rig platforms, satellites, etc.) are both mission and performance effective and affordable. The response to the challenge involves three principal parts:

- The first part is an organization of the system data through an integrated multi-domain data schema for representing system product and process data. This will be essential to developing and operating an advanced integrated environment.

- The second is an integrated environment that employs formal methods and automation to support the full range of data manipulation and communication required by complex system life cycle activities. This environment enables a broad spectrum of life cycle participants to evaluate alternatives in multiple domains simultaneously; provides a way for stakeholders to understand their needs in relation to the enterprise as a whole; and provides a continuous proactive means of identifying and successfully addressing key challenges for a complex system over time.

- The third is an evolved culture where enterprise-wide cooperation is the rule and individual contributions are encouraged and efficiently managed.

Working within an integrated environment based on these three elements will provide a common frame of reference in which sophisticated relationships among technical domains, and between these domains and a system's affordability, can be explicitly identified and analyzed. The key to realizing gains from the combined elements is the data schema, essential to integrating 
the disparate data sets in use by complex system program offices and other related enterprises.

The MV Program needs to stay abreast of evolving data transfer standards to gain leverage by building on their standardized business processes, data type schemas, data dictionaries, and publish-and-subscribe message techniques wherever feasible. The MV common data schema uses, rather than competes with, these other data transfer and data storage means of achieving interoperability.

The MV response to the challenge involves the analysis and assessment of two primary concepts, each to be investigated against an evolving set of applicable schema requirements employing the primary systems engineering process described in ANSI/EIA 632, (1999). These two concepts are:

1. An integrated multi-domain data schema for representing system product and process data. In this concept "data schema" means a depiction of the structure and constraints of the contents of an information system: a data model, which defines a vocabulary of terms, their properties and relationships, and how the information to which they refer must be organized.

2. Enhancement of COTS integrated ERP (Enterprise Resource Planning) systems already in use in multiple Aerospace and Defense companies and planned for several DOD military services and the Defense Logistics Agency (DLA).

Concept (1) requires the development of a data schema constructed from existing or evolving standards that can completely represent all required product and process data. Such standards include the IGES and STEP standards as examples of a partially complete standard set. Concept (2) currently requires the migration of legacy databases to an applicable Meta database in accordance with the database format selected by each ERP provider.

The MV approach contrasts with the current object-oriented interoperability approach employing middleware that does not necessitate the use of the data schema concept (1) but which requires an integration schema for integration of the various databases to an Application Architectural Framework.

All of the above concepts must be traded off against an evolving set of MV program requirements, particularly those of a derived nature. These are evolving because the cost and schedule of the ultimate solution must be timely and affordable and as such, each requirement must be examined for its meaningful application to the MV program.

An extensive initial set of requirements have been evolved starting with the MV Statement of Work, with additional requirements derived from the MV Concept of Operations, decomposition of associated "Use Cases," user interviews, DOD Instruction 5000.2, development of existing relevant stan- 
dards activities and initiatives, and commercial enterprise resource planning and management systems. The requirements defined to date address the schema integration Framework, Schema, and Life Cycle User Information System Requirements. The first demonstration of the MV schema was successfully conducted in July 2001 in the PM Abrams pilot program.

In order to reduce the time necessary to develop the schema, avoid duplication of effort, and enhance industry acceptance and use, the MV team agreed on a strategy of reusing existing schemas that cover some portions of the MV scope rather than developing the MV data schema from scratch. Such reuse will take two forms: integration into the actual MV schema, and reference from the MV schema.

Promulgation of the MV schema as a standard is essential for its acceptance and adoption for use by both industry and the DOD. A MV Schema Transition Strategy was developed in February 2002 defining how the MV schema can be transitioned to a formal living standard that is sought-after, endorsed, broadly accepted, and maintained and used by industry and DOD.

\section{CURRENT STATUS}

Any Government or commercial organization faces a dilemma in determining the degree of support to which one commits to achieve their organization's interoperability objectives. The complexity of the organization, complexity of product and/or services, present degree of product and process information systems, subsystems, and life cycle domains interoperability all dictate a different interoperability strategy and business case. What is important though is to deploy a structured business case methodology to guide one to make the most cost effective interoperability implementation decisions.

The primary issue is that there are many focused application solutions that are addressing a piece of the total interoperability need. These efforts provide partial solutions to pieces of the virtual enterprise-wide interoperability objective for particular areas of the enterprise. These activities do not provide the integration or framework for the entire enterprise life cycle. The MV Program is the first top down systems engineering approach that views all of the technical and business complex systems life cycle data requirements in a virtual enterprise-wide environment. MV provides the framework that builds on many of today's partial integration solutions. The MV Program integrates data schemas of these partial solutions with a MV top-level schema. The MV Program technical approach is object-flavored, modeled in EXPRESS, utilizes the ISO 15926, (2001) as the integration model, builds on ISO 10303, (1999) STEP APs, and has integrated with a portion of the U.S. Department of Defense Data Architecture. Two of the most significant 
integration activities in the ISO standards community are the IIDEAS (Integration of Industrial Data for Exchange, Access, and Sharing) project sponsored by the ISO TC184 SC4 activity (ISO IS 18876, 2000) and Product Life Cycle Support (PLCS, 1999). IIDEAS provides the underpinnings of the integration approach that the MV Program has developed. The MV Program is working with the STEP and PLCS standards activities to incorporate their work into the MV schema.

In addition, the commercial world is moving towards implementation of XML (2000, 2001), SOAP (2000), and other standards for World Wide Web data transfer and application integration architecture middleware, as well as deploying extensive ERP systems. MV will augment their successful implementation.

In the first year, the MV Program built an Interoperability Testbed to validate the schema, framework, and translators for the Abrams Tank pilot. This effort will be followed with additional DOD complex systems pilot application validations that will be selected by the MV stakeholders. A draft ANSI/EIA standard will be prepared and implemented that will subsequently be transitioned to an ISO standard. On 5/12/02 the Government Electronics \& Information Technology Association (GEIA) issued a press release announcing the formation of a new standardization project based on the MV Program. The project, designated as GEIA/EIA-927 Common Data Schema for Complex Systems, will involve both industry and Government experts. In subsequent years a not-for-profit consortium will be formed to promulgate and maintain the use of the MV schema standard.

\section{REFERENCES}

ANSA/EIA 632, (1999), Processes for Engineering a System, http://global.ihs.com/ ANSI X12 EDI, (2000), Electronic Data Interchange.

ISO IS 10303, (1999), Product data representation and exchange STEP, TC 184 SC4. ISO 15926 EPISTLE Core Model 3.1, (2001), Integration of Life-Cycle Data for Oil \& Gas production facilities.

ISO IS 18876, (2000), Integration of Industrial Data for Exchange, Access, and Sharing, TC 184 SC4.

OMG-CORBA-2, (1999), Common Object Request Broker Architecture- Interoperability. PLCS, (1999), Product Life Cycle Support, planned submission to ISO TC184 SC4 in 2002. SOAP 1.1, (2000), Simple Object Access Protocol.

XML, REC-xml-20001006, (2000), Extensible Markup Language (XML), 1.0 Second Edition - W3C Recommendation 6 October.

XML, REC-xmlschema-1/2-20010502, (2001), XML Schema Part 1/2: Structures/Data types, - W3C Recommendation 02 May. 